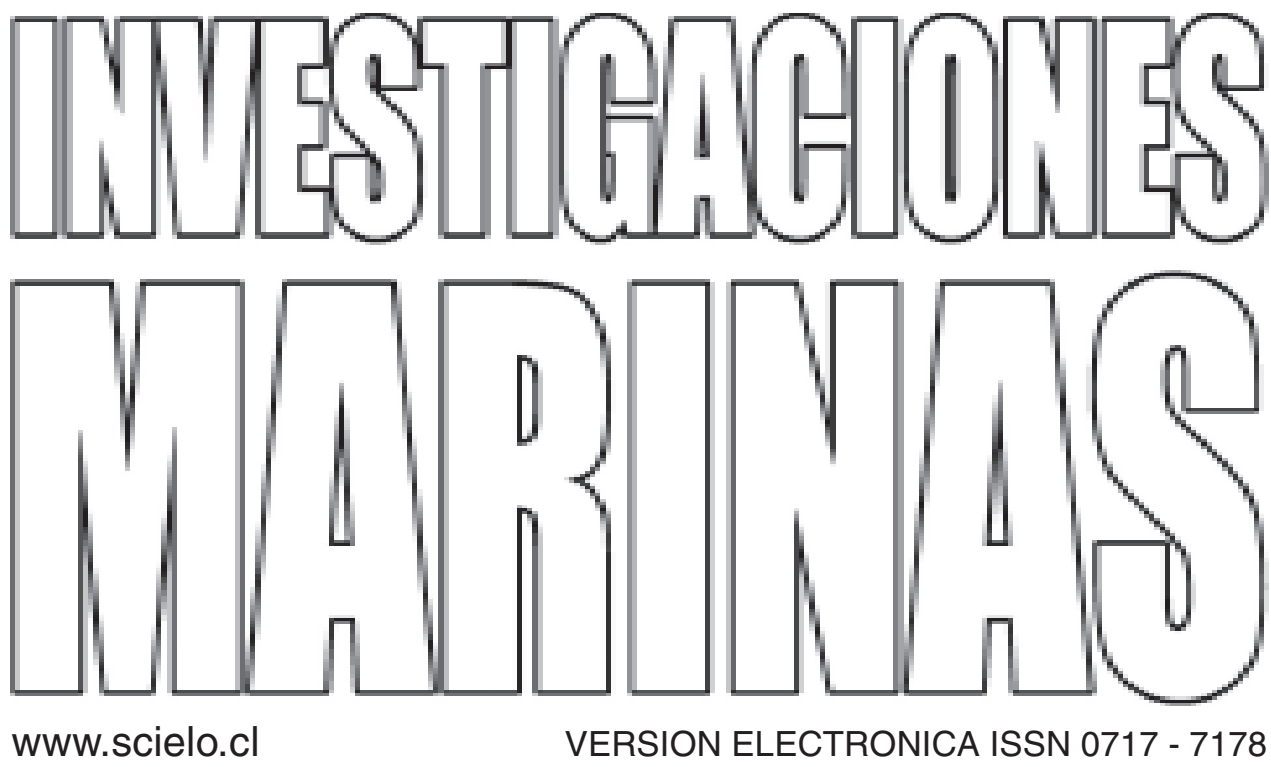

INVESTIGACIONES MARINAS es una revista que considera para su publicación los resultados de investigaciones científicas y tecnológicas originales en ciencias del mar, dando preferencia a los trabajos realizados en el Océano Pacífico Sudoriental y Océano Austral. Los artículos y notas pueden ser publicados en idioma español o inglés.

La revista es de periodicidad semestral y es editada por la Escuela de Ciencias del Mar de la Facultad de Recursos Naturales de la Pontificia Universidad Católica de Valparaíso.

INVESTIGACIONES MARINAS is a journal for the purpose of publishing results either from scientific or technological research in marine science. The journal will give priority to those manuscripts referring to the Southeastern Pacific or the Southern Ocean. Submission can be made in Spanish or English.

The journal is edited by Escuela de Ciencias del Mar, Facultad de Recursos Naturales, Pontificia Universidad Católica de Valparaíso, and published twice a year.

PONTIFICIA UNIVERSIDAD CATÓLICA DE VALPARAÍSO

FACULTAD DE RECURSOS NATURALES

ESCUELA DE CIENCIAS DEL MAR 


\section{EVALUADORES DE LOS TRABAJOS INCLUIDOS EN ESTE NÚMERO}

\author{
Hugo Arancibia \\ Universidad de Concepción \\ Roberto Bahamonde \\ Instituto de Fomento Pesquero \\ María Angela Barbieri \\ Pontificia Universidad Católica de Valparaíso \\ José Luis Brito \\ Museo Municipal de Ciencias Naturales y \\ Arqueología de San Antonio \\ Cristian Canales \\ Instituto de Fomento Pesquero \\ Enrique Dupré \\ Universidad Católica del Norte \\ Dante Figueroa \\ Universidad de Concepción \\ Ismael Kong \\ Universidad de Antofagasta \\ Roberto Meléndez \\ Museo Nacional de Historia Natural \\ Germán Pequeño \\ Universidad Austral de Chile \\ Marco Antonio Retamal \\ Universidad de Concepción \\ Hellmuth Sievers \\ Universidad de Valparaíso
}

\author{
Pedro Báez \\ Museo Nacional de Historia Natural \\ Fernando Balbontín \\ Universidad de Valparaíso \\ Arnaud Bertrand \\ Institut de Recherche pour le Developpement \\ Mario Cáceres \\ Servicio Hidrográfico y Oceanográfico de la \\ Armada \\ Leonardo Castro \\ Universidad de Concepción \\ Fernando Espíndola \\ Universidad de Concepción \\ Pierre Freón \\ Institut de Recherche pour le Developpement \\ Julio Lamilla \\ Universidad Austral de Chile \\ Patricio Pavez \\ Pontificia Universidad Católica de Valparaíso \\ Renato Quiñones \\ Universidad de Concepción \\ Luciano Rodríguez \\ Universidad del Mar \\ Ingo Wehrtmann \\ Universidad de Costa Rica
}

Eleuterio Yáñez

Pontificia Universidad Católica de Valparaíso 


\title{
Clave taxonómica para el reconocimiento de especies de rayas chilenas (Chondrichthyes, Batoidei)
}

\author{
Julio Lamilla ${ }^{1} \&$ Sylvia Sáez ${ }^{2}$ \\ ${ }^{1}$ Programa Doctorado en Ciencias Biológicas, Universidad de Concepción \\ ${ }^{1,2}$ Instituto de Zoología "Ernst F. Kilian”, Universidad Austral de Chile, Casilla 567, Valdivia, Chile \\ e-mail: jlamilla@uach.cl
}

RESUMEN. Se presenta una clave para reconocer especies del Superorden Batoidea de Chile, agrupadas en las familias Rhinobatidae, Pseudorajidae, Rajidae, Narcidae, Torpedinidae, Dasyatidae, Urolophidae, Myliobatidae y Mobulidae. Se indica la distribución geográfica de cada una de las 38 especies consideradas.

Palabras claves: taxonomía, rayas, distribución geográfica, Chile.

\section{Taxonomic key for the identification of Chilean rays and skates species (Chondrichthyes, Batoidei)}

\begin{abstract}
A taxonomic key to identify fish species of the Chilean Superorden Batoidea, grouped in the families: Rhinobatidae, Pseudorajidae, Rajidae, Narcidae, Torpedinidae, Dasyatidae, Urolophidae, Myliobatidae and Mobulidae is presented. For the currently 38 batoid species considered, the geographic distribution is indicated.
\end{abstract}

Key words: taxonomy, skates, geographic distribution, Chile.

\section{INTRODUCCIÓN}

Los Batoideos en el mundo han sufrido drásticos cambios en su taxonomía en los últimos decenios, en especial desde el momento en que empezaron a ser utilizados elementos de la morfología interna como: el neurocráneo (Hulley, 1970, 1972; McEachran, 1982a, 1982b, 1983; Stehmann, 1970); el escápulo-coracoides (McEachran \& Compagno, 1979, 1982; McEachran, 1982a, 1983) y como carácter específico los órganos copuladores (Hulley, 1970, 1972; Ishiyama \& Hubbs, 1968; McEachran 1982a, 1982b, 1983; Stehmann, 1970).

Los cambios más notables han ocurrido en la antigua familia Rajidae y menores en otras familias; esta situación no ha sido ajena a Chile, para el cual se entrega a continuación, un resumen de los cambios más importantes.

Bahamonde \& Pequeño (1975) señalan la pre- sencia de tres géneros de Rajidae: Breviraja, Psammobatis y Raja con ocho especies. Luego, McEachran (1982a) reubica a Psammobatis lima Poeppig, 1835 y a Psammobatis brevicaudatus Cope, 1877 en el género Sympterygia e incorpora a Psammobatis caudispina Hildebrand, 1946 a la sinonomia de Psammobatis brevicaudatus. Después, McEachran (1983) revisando material argentino, revalida a Psammobatis rudis Günther, 1870 de la sinonimia de Psammobatis scobina (Philippi, 1901).

McEachran \& Miyake (1984) reubican a Breviraja longicauda De Buen, 1959 como Bathyraja longicauda (De Buen, 1959) y a Breviraja nigerrima De Buen, 1959 como Malacoraja nigerrima De Buen, 1959 respectivamente. Además, creyendo extraviado el holotipo de esta última especie, designaron un neotipo.

McEachran (1984) e Ishihara \& Ishiyama (1986) consideran que dentro del Suborden Rajoidei hay 
dos familias, y 29 géneros y subgéneros.

Pequeño \& Lamilla (1985) estudiando una colección de rayas del sur del país, reubican especies del género Raja en el género Bathyraja, adicionan a Breviraja macloviana Norman, 1937 y confirman la revalidación de Psammobatis rudis Günther, 1870.

Parin \& Kotlyar (1985) describen dos nuevas especies de rayas eléctricas para la elevación de Nazca, Torpedo microdiscus y T. semipelagica.

Lamilla (1986) señala a Bathyraja albomaculata como primer registro para la costa chilena.

Leible \& Stehmann (1987) reportan para aguas chilenas a Raja (Dipturus) trachyderma Krefft \& Stehmann, 1975 (= Dipturus trachyderma). Luego, Leible (1987) describe juveniles y adultos de Raja (Dipturus) flavirostris Guichenot, 1848 (=Dipturus chilensis), extendiendo esta sinonimia debido a la prioridad de esta última sobre $R$. (Dipturus) flavirostris (Pequeño, 1989).

Lamilla \& Meléndez (1989) comunican la presencia de Dasyatis violacea (Bonaparte, 1832) en las cercanías de isla de Pascua.

McEachran \& Miyake (1990) excluyen a Malacoraja nigerrima del género Malacoraja y la reubican en Raja subgénero Rajella.

McEachran \& Miyake (1990) postulan que se agruparía a nivel de familia a los taxa que forman el Grupo I propuesto por ellos, coincidente con la familia Pseudorajidae sugerida por Ishihara (1990) que reuniría entre otros, a los géneros Bathyraja, Psammobatis y Sympterygia.

Otro cambio importante ocurre con Gurgesiella furvescens De Buen, 1960 para la zona de Valparaíso, originalmente en una familia monotípica (Gurgesiellidae De Buen, 1960), reconocida por Hulley (1972); después asignada a la familia Pseudorajidae por McEachran \& Compagno (1979) y últimamente, incluida en el Grupo II (= Familia Rajidae de Ishihara, 1990) según la proposición de McEachran (1984) y McEachran \& Miyake (1990).

Pequeño \& Lamilla (1993) comparan la fauna de batoídeos de Chile y Argentina-Uruguay sobre la base de antecedentes publicados y nuevos hallazgos, aumentando de 26 especies (Pequeño, 1989) a 35 especies chilenas.

Lamilla et al. (1995) incorporan la presencia de Dasyatis brevis (Garman, 1913) para el norte de Chile. Pequeño \& Lamilla (1996) comunican la presencia de Sympterygia bonapartii (Müller \& Henle, 1841) en el estrecho de Magallanes. Años después,
Lamilla \& Pequeño (1999) señalan a Bathyraja multispinis (Norman, 1937) en aguas chilenas.

McEachran \& Dunn (1998) proponen que los subgéneros creados por Stehmann (1970) para la familia Rajidae, sean elevados a la categoría de géneros.

En esta reseña, debería incorporarse además de los ejemplares anteriormente descritos, a la raya antártica Amblyraja georgiana (Stehmann, 1985), colectada alrededor de la península Antártica y Amblyraja doello-juradoi Pozzi, 1935 en el estrecho de Magallanes.

Con respecto a las familias Rhinobatidae y Urolophidae, la situación en géneros y especies es similar desde Bahamonde \& Pequeño (1975). Cabe señalar la situación confusa que se ha mantenido por años respecto a Tarsistes philippi Jordan, 1918; debido a que el holotipo jamás ha sido estudiado ni descrito, con ello Rhinobatidae se mantiene actualmente con una sola especie (Pequeño, 1989; Eschmayer, 1998). En la familia Mobulidae, Notarbartolo di Sciara (1987) sinonimiza Mobula lucasana Beebe \& Tee-Van, 1941 como Mobula thurstoni (Lloyd, 1908). De acuerdo a este autor y a Chirichigno (1998), se reconoce a esta especie en la familia Mobulidae, y no incluida en Myliobatidae como lo propone Eschmayer (1998).

Los cambios anteriormente citados en la literatura científica han estado ajenos a la comunidad científica nacional y difícilmente, se podría decir que actualmente se reconocen 38 especies de batoídeos en aguas chilenas, las cuales se indican a continuación.

\section{ESPECIES DEL SUPERORDEN BATOIDEA EN AGUAS CHILENAS}

(entre paréntesis se indica el número de especies de cada familia)

FAMILIA RHINOBATIDAE (1)

Rhinobatos planiceps Garman, 1880

FAMILIA PSEUDORAJIDAE (17)

Bathyraja albomaculata (Norman, 1937)

Bathyraja brachyurops (Fowler, 1910)

Bathyraja eatonii (Günther, 1876)

Bathyraja griseocauda (Norman, 1937)

Bathyraja longicauda (De Buen, 1959)

Bathyraja maccaini Springer, 1971

Bathyraja macloviana (Norman, 1937)

Bathyraja magellanica (Philippi, 1901) 
Bathyraja multispinis (Norman, 1937)

Bathyraja peruana McEachran \& Miyake, 1984

Bathyraja schroederi Krefft, 1968

Psammobatis scobina (Philippi, 1857)

Psammobatis normani McEachran, 1983

Psammobatis rudis Günther, 1870

Sympterygia brevicaudata (Cope, 1877)

Sympterygia bonapartii Müller \& Henle, 1841

Sympterygia lima (Poeppig, 1835)

FAMILIA RAJIDAE (8)

Gurgesiella furvescens De Buen, 1959

Amblyraja doello-juradoi Pozzi, 1935

Amblyraja frerichsi Krefft, 1968

Amblyraja georgiana Norman, 1938

Dipturus chilensis Guichenot, 1848

Dipturus trachyderma Krefft \& Stehmann, 1975

Rajella nigerrima (De Buen, 1960)

Rajella sadowskii Krefft \& Stehmann, 1974

FAMILIA NARCINIDAE (1)

Discopyge tschudii Haeckel, 1845

FAMILIA TORPEDINIDAE (3)

Torpedo tremens De Buen, 1959

Torpedo microdiscus (Parin \& Kotlyar, 1985)

Torpedo semipelagica (Parin \& Kotlyar, 1985)

FAMILIA DASYATIDAE (2)

Dasyatis violacea (Bonaparte, 1832)

Dasyatis brevis (Garman, 1913)

FAMILIA UROLOPHIDAE (2)

Urobatis marmoratus (Philippi, 1892)

Urotrygon chilensis (Günther, 1871)

FAMILIA MYLIOBATIDAE (2)

Myliobatis chilensis (Philippi, 1892)

Myliobatis peruvianus Garman, 1913

FAMILIA MOBULIDAE (2)

Mobula thurstoni (Lloyd, 1908)

Mobula tarapacana (Philippi, 1892)

Es importante destacar, que con posterioridad a la publicación de la obra "Vida de los peces en aguas chilenas" de Mann (1954), que incluye una clave de rayas para ocho especies y dos géneros, no se ha publicado nada similar, que permita actualizar los cambios taxonómicos experimentados por este grupo de peces. Para suplir esta falencia, en este trabajo se entrega una clave actualizada de las modificacio- nes taxonómicas del Superorden Batoidea y de los caracteres considerados para el reconocimiento taxonómico sus especies. No se incluye la sinonimia por estar mencionada en la literatura específica.

\section{MATERIALES Y MÉTODOS}

Con la finalidad de comparar los caracteres diagnósticos para géneros, subgéneros y especies, se consideraron las descripciones de los siguientes trabajos: Beebe \& Tee-Van (1941), Bigelow \& Schroeder (1965), Chirichigno (1969, 1974, 1978, 1998), Chirichigno \& McEachran (1979), Chu et al. (1981), Compagno (1973, 1977), De Buen (1959a, 1959b, 1959c, 1960), Fowler (1945), Guichenot (1848), Henríquez et al. (1981), Hubbs \& Ishiyama (1968), Hulley \& Stehmann (1977), Hureau (1966), Ishiyama (1958), Ishiyama \& Hubbs (1968), Krefft (1968), Lamilla (1986), Lamilla et al. (1984, 1995), Lamilla \& Meléndez (1989), Leible (1987, 1988), Leible \& Stehmann (1987), Leible et al. (1990), Mann (1954), McEachran (1982a, 1982b, 1983, 1984, 1985), McEachran \& Compagno (1982), McEachran \& Matheseon (1985), McEachran \& Miyake (1986, 1987), McEachran \& Dunn (1998), Menni (1971, 1972a, 1972b, 1973), Nelson (1994), Nishida (1990), Norman (1935, 1937), Notarbartolo di Sciara (1987), Oliver (1943), Parin et al. (1980), Pequeño (1975a, 1975b, 1989, 1997), Pequeño \& Lamilla (1979, 1993, 1996), Philippi (1857a y 1857b), Springer (1971), Stehmann (1970, 1979, 1985, 1986) y Zorzi \& Anderson (1988).

Se prepararon ejemplares de Dipturus chilensis, Psammobatis normani, Psammobatis scobina, Psammobatis rudis, Sympterygia lima, Bathyraja albomaculata, Bathyraja brachyurops y Bathyraja multispinis, cuyas partes carnosas fueron previamente maceradas en solución de $\mathrm{NaOH}$ al $10 \%$, para ser comparadas con las descripciones mencionadas, y en algunos casos se utilizaron radiografías.

El autor principal revisó material biológico de las colecciones del Instituto de Zoología Universidad Austral de Chile (IZUA), de la Universidad de Concepción y del Museo Nacional de Historia Natural de Santiago.

Para facilitar el reconocimiento y definir ciertos caracteres diagnósticos presentes en la Clave de reconocimiento, se entrega un esquema general con la morfología externa de una raya tipo (Fig. 1), ilustrando algunos caracteres diagnósticos de algunas especies y familias. Estos esquemas están comple- 
mentados por un breve glosario con los términos más especializados utilizados en la Clave de reconocimiento.

\section{Clave para familias y especies chilenas del Superorden Batoidei}

1 (2) Aletas pectorales no fusionadas a la cabeza no forman un disco completo, cuerpo como tiburón, hocico alargado..

RHINOBATIDAE

- Disco subtriangular amplio en su parte posterior, aguzado hacia delante (Fig. 2a). Cuerpo, cabeza y cola deprimida más larga que el cuerpo, aleta caudal pequeña, su porción subcaudal es débil y sin lóbulo (Islas Galápagos; Perú: Punta Pizarro; Chile: Arica, en Hornitos y La Portada en Antofagasta) .. Rhinobatos planiceps Garman, 1880

2 (1) Aletas pectorales fusionadas a la cabeza for- man un disco completo, cuerpo deprimido 3

3 (4) Presencia de grandes órganos eléctricos a cada lado de la cabeza (Fig. 2b), tallo corto, aleta caudal grande.

4 (3) Ausencia de órganos eléctricos a cada lado de la cabeza, tallo fino a delgado, aleta caudal pequeña

5 (6) Con dos aletas dorsales ............................... 7

6 (5) Con una aleta dorsal o ausencia de ella .... 11

7 (8) Rostro flexible y débil; los radios de la aleta pectoral alcanzan el extremo distal del rostro..

PSEUDORAJIDAE ....17

8 (7) Rostro rígido y fuerte, los radios de la aleta pectoral no alcanzan el extremo distal del rostro..

. RAJIDAE....49
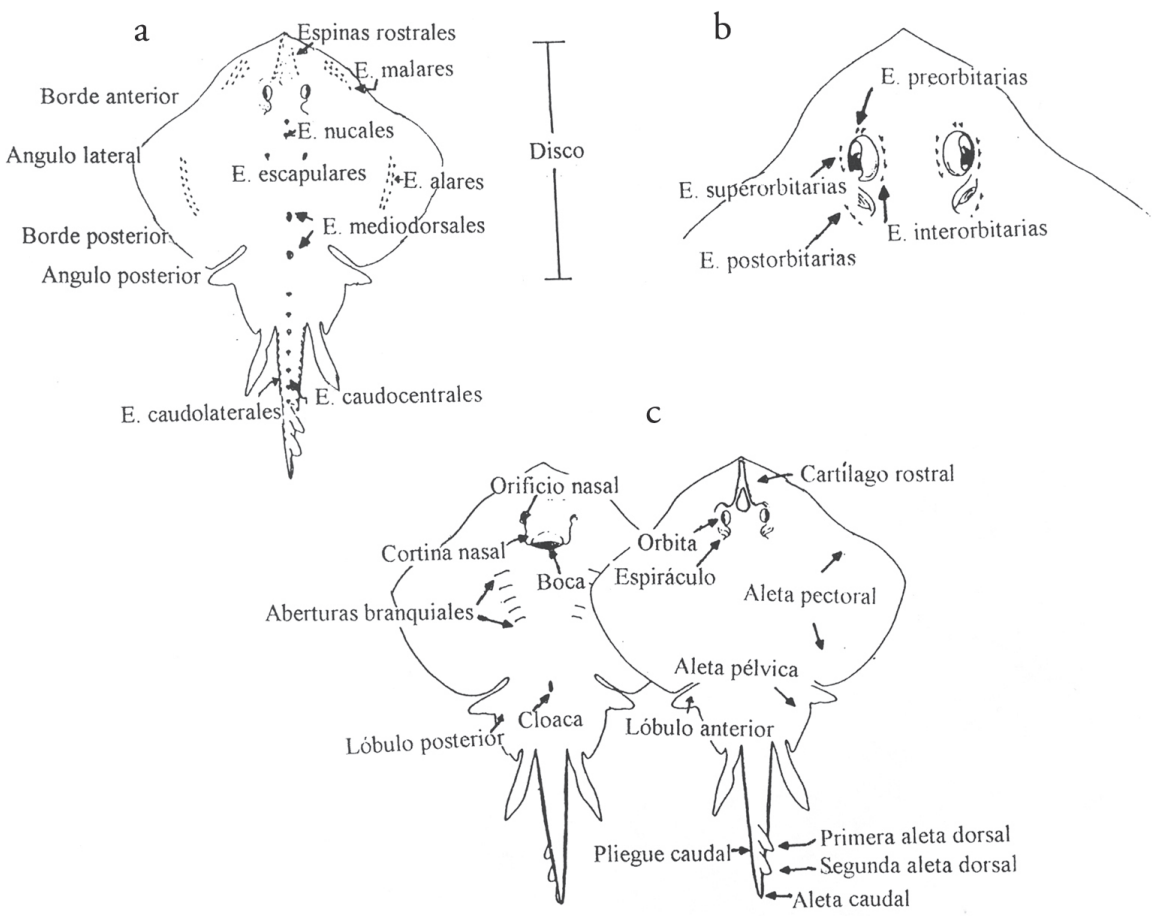

Figura 1. a) Vista dorsal de la morfología externa y nomenclatura de la espinulación en una raya tipo (modificado de Chirichigno, 1998), b) vista dorsal del detalle de la espinulación orbital (modificado de Chirichigno, 1998), c) morfología externa (dorsal y ventral) de una raya tipo (modificado de Chirichigno, 1998).

Figure 1. a) Dorsal view of external morphology and squamation nomenclature in a general skate (modified from Chirichigno, 1998), b) dorsal view of orbital squamation detail (modified from Chirichigno, 1998), c) external morphology (dorsal and ventral) in a general skate (modified from Chirichigno, 1998). 
9 (10) Boca pequeña y transversal, cortina nasal estrecha y grande; espiráculos inmediatamente detrás de los ojos.

NARKIDAE

- Disco aproximadamente circular. Boca inferior pequeña, protráctil, con dientes en placas dentarias cortas, en bandas en mosaico estrechas e inclinadas, pequeños y aplastados, con sus márgenes interiores agudos y angulados (Perú: de Huacho a Chancay; Chile: Coquimbo, Pichidangui, Caleta La Ligua, Ancud y Corcovado; Argentina y Uruguay) Discopyge tschudii Haeckel, 1845.

10 (9) Boca grande y ampliamente arqueada, cortina nasal amplia y corta; espiráculos a corta distancia por detrás de los ojos. ..... TORPEDINAE...63

11 (12) Cabeza forma parte del disco, sin aletas dorsales 13

12 (11) Cabeza marcadamente fuera del disco, una aleta dorsal 15

13 (14) Disco más amplio que largo, cola variable: delgada como látigo o firme y más corta que el disco .....

. DASYATIDAE...67

14 (13) Disco igual o más largo que amplio, cola corta pero más larga que el disco.

UROLOPHIDAE...69

15 (16) Cabeza y hocico se diferencian notoriamente del resto del disco (Fig. 2c); el hocico forma un simple lóbulo o un par de amplios lóbulos redondeados; sin aleta cefálica; dientes en pavimento.. .

MYLIOBATIDAE...71

16 (15) Cabeza (en frente de los espiráculos) notoriamente marcada del resto del disco; presencia de un par de elongadas aletas cefálicas (Fig. 2d); dientes con pequeñas cúspides .... MOBULIDAE....73

17 (18) Ojos relativamente grandes a moderados, el diámetro orbital menos de 1,4 veces en el espacio interorbital. 19

18 (17) Ojos pequeños, el diámetro orbital 1,4 a 4 veces en el espacio interorbital 23

19 (20) Tallo relativamente amplio en la base, generalmente igual o más grande que el ancho interorbital; las aletas dorsales confluyen; sin espina interdorsal. Disco con dentículos, pero carente de aguijones (Chile: de Arica a Constitución y de Corral a Ancud) Sympterygia lima (Poeppig, 1835).

20 (19) Tallo relativamente estrecho en la base, generalmente menor que el ancho interorbital; las aletas dorsales no confluyen; con un aguijón interdorsal.
Disco en juveniles grandes y adultos generalmente con aguijón nucal y varios en una serie media .. 21

21 (22) Bordes anteriores del disco ampliamente redondeados y poco ondulados. Serie media de aguijones sobre el disco incompleta; sin aguijones sobre la región nucal, prenucal y en el origen de la cintura pélvica; aletas dorsales con un aguijón entre ellas. Superficie dorsal del disco café a gris con manchas oscuras a claras esparcidas sobre el disco (Desde Ecuador hasta Chile central).... Sympterygia brevicaudata (Cope, 1877).

22 (21) Bordes anteriores del disco prácticamente rectos o sólo ligeramente ondulados. Serie media de aguijones sobre el disco ausente o presente por detrás de la región nucal y varios sobre la base de las aletas pélvicas. Superficie dorsal del disco café con manchas oscuras sobre el hocico y dispuestas simétricamente sobre el disco (Chile: estrecho de Magallanes; Argentina: Rawson; Uruguay; sur de Brasil) ... Sympterygia bonapartii Müller \& Henle, 1841.

23 (24) Disco con el margen anterior convexo desde la punta del hocico hasta el nivel de las órbitas. Cartílago rostral no unido al cráneo; con fontanela nasal 25

24 (23) Disco con el margen anterior cóncavo desde la punta del rostro hocico hasta algo más arriba del nivel de las órbitas. Cartílago rostral unido al cráneo; sin fontanela nasal 29

25 (26) Disco con el margen anterior convexo desde la punta del rostro al nivel de las órbitas, fuertemente cóncavo desde el nivel de las órbitas al nivel de los espiráculos y ampliamente redondeado posterior a ellos. Fontanela anterior más bien esférica. Con 3 a 5 filas irregulares de aguijones sobre la región caudal (Chile: isla Guafo hasta el sur de Argentina, Bahía Blanca e islas Malvinas; Uruguay) Psammobatis normani McEachran, 1983.

26 (25) Disco con el margen anterior convexo, desde la punta del rostro hasta el nivel de los espiráculos, para luego continuar de forma redondeada. Fontanela anterior, más bien alargada. Con 1- 5 filas irregulares de aguijones sobre la región caudal

27 (28) Superficie dorsal cubierta de dentículos dérmicos y con aguijones que forman un semicírculo sobre el margen interno de la órbita. Dorsalmente la región caudal presenta una fila irregular de aguijones (Chile: isla Guafo; Argentina: isla Los Esta- 
dos y Puerto Deseado; Uruguay) Psammobatis rudis Günther, 1870.

28 (27) Superficie dorsal del disco lisa, a excepción de la región medio dorsal debido a la presencia de numerosas pequeñas denticulaciones. Dorsalmente la región caudal presenta 3 a 5 filas irregulares de aguijones (Chile: punta Camarones, Taltal, caleta La Ligua, Corral hasta Cabo de Hornos; Argentina; Uruguay). .. Psammobatis scobina (Philippi, 1857).

29 (30) Disco con aguijones 31

30 (29) Disco sin aguijones .45

31 (32) Superficie del disco siempre lisa. Con más de 40 aguijones en la línea media dorsal, ubicados desde la región nucal hasta el origen de la primera aleta dorsal. Café grisáceo con trazas de manchas pálidas y oscuras desvanecidas (Chile: canal Darwin al estrecho de Magallanes; Argentina: isla Los Estados a islas Malvinas; Uruguay) Bathyraja multispinis (Norman, 1937).

32 (31) Superficie del disco con espinulación variable. Con menos de 30 aguijones en la línea media dorsal .33

33 (34) De 25 a 30 aguijones en la serie media dorsal. .35

34 (33) Menos de 25 aguijones en la serie media dorsal 39

35 (36) Dorsal y ventralmente de coloración similar, siendo el vientre de color café uniforme incluida región pélvica y caudal, pequeñas manchas negras en la región nucal y comienzos del dorso; 26 a 30 aguijones extendidos desde el nivel del ancho máximo del disco, hasta el origen de la primera aleta dorsal (Desde Perú hasta Constitución, Chile) .. Bathyraja longicauda (De Buen, 1959).

36 (35) Dorsal y ventralmente de coloración diferente, siendo el vientre de color blanco amarillento; 25 a 30 aguijones extendidos desde la región nucal, hasta el origen de la primera aleta dorsal ............ 37

37 (38) El ano más cerca del extremo de la cola que la punta del hocico. Hocico, aproximadamente un $20 \%$ en el ancho del disco; el ancho del disco 3/4 de la longitud total (Chile: isla de Chiloé y estrecho de Magallanes; Argentina: Patagonia e islas Malvinas; Uruguay)

Bathyraja magellanica (Philippi, 1901).

38 (37) El ano más o menos equidistante de la pun- ta del hocico al extremo de la cola. Hocico entre un $14 \%$ a $17 \%$ en el ancho del disco; el ancho del disco menor que 3/4 de la longitud total (Chile: canal Concepción, estrecho de Magallanes y cabo de Hornos; Argentina: Patagonia e Islas Malvinas; Uruguay)

Bathyraja macloviana (Norman, 1937).

39 (40) Disco con pequeñas manchas blancas redondeadas; 17 a 23 aguijones en la línea media dorsal. Color café oscuro con pequeñas manchas blancas redondas, a veces con márgenes más oscuros. Ventralmente de color blanco, excepto los márgenes posteriores de las aletas pectorales y gran parte de la cola (Chile: isla Guafo e isla Guamblin; Argentina: Patagonia e islas Malvinas; Uruguay) ..... Bathyraja albomaculata (Norman, 1937).

40 (39) Disco sin manchas blancas redondeadas; 8 a 18 aguijones en la línea media dorsal .............. 41

41 (42) Presencia de aguijones escapulares y orbitales; 8 a 15 aguijones mediales sobre la cola (Circumantártica, desde islas Orcadas y Shetland del Sur a la península Antártica)

Bathyraja maccaini Springer, 1971.

42 (41) Ausencia de aguijones escapulares y orbitales; 8-18 aguijones en la línea media dorsal.

43 (44) Hocico relativamente largo, la longitud preorbital más del $25 \%$ en el ancho del disco; 8 a 18 aguijones mediales sobre la cola (Circumantártica, desde las islas Kerguelen, Shetland del Sur, Orcadas del Sur hasta península Antártica)

Bathyraja eatonii (Günther, 1876).

44 (43) Hocico más corto, la longitud preorbital menos del 25\% en el ancho del disco; 12 a 18 aguijones en una serie media del disco y cola, o solo sobre la cola (Chile: Valdivia, canal Concepción, estrecho de Magallanes e isla Diego Ramírez; Argentina: Patagonia e islas Malvinas; Uruguay) ..... . Bathyraja brachyurops (Fowler, 1910).

45 (46) Cuerpo dorsalmente gris y ventralmente gris o blanco amarillento 47

46 (45) Cuerpo dorsalmente y ventralmente café oscuro. Hocico alargado, levemente encorvado hacia arriba, con punta roma. Numerosas espínulas distribuidas sobre el disco; vientre liso; mandíbula casi recta; 24 aguijones caudocentrales; ambas aletas dorsales separadas en las bases; flecos de las fosas nasales, mandíbulas y borde de la cloaca, blancos 
(Chile: isla Mocha; Argentina: frente a bahía de Samborombón; Uruguay)

$$
\text { Bathyraja schroederi Krefft, } 1968 .
$$

47 (48) Ventralmente blanco amarillento, con excepción de la cara ventral de la región caudal que es siempre de color gris; 15 a 19 aguijones caudocentrales. El ano más cerca de la punta del hocico que del extremo de la cola (Chile: bahía San Pedro a Península Taitao; Argentina: Patagonia, islas Malvinas, islas Shetland del Sur; Uruguay)..... Bathyraja griseocauda (Norman, 1937).

48 (47) Ventralmente de color gris parduzco; 18 a 26 aguijones caudocentrales. El ano equidistante de la punta del hocico y del extremo de la cola (Desde el sur de Ecuador hasta Iquique en Chile; Argentina; Uruguay)

Bathyraja peruana McEachran \& Miyake, 1984.

49 (50) Superficie ventral de la cola, con excepción de una estrecha línea media, cubierta uniformemente con dentículos dérmicos; un parche triangular de 11 aguijones sobre la región nucal y escapular. Uniformemente café oscuro, excepto las aletas dorsales que son de color café negruzco (Sur del Perú; Chile: Valparaíso y archipiélago de los Chonos) Rajella nigerrima (De Buen, 1960).

50 (49) Superficie ventral de la cola, no presenta dentículos dérmicos, o si los presenta, estos se distribuyen irregularmente; sin parche triangular de aguijones sobre la región nucal y escapular ......51

51 (52) Disco romboide, hocico largo .53

52 (51) Disco notablemente más ancho que largo, extremo del hocico no es saliente, existe apenas una corta punta triangular y roma. Ano más cerca del extremo del hocico que de la cola (Desde islas Galápagos, Callao, Perú hasta Los Vilos y Papudo, Chile) ...... Gurgesiella furvescens De Buen, 1959.

53 (54) Aguijones nucales presentes, generalmente 1 ó 2 .55

54 (53) Aguijones nucales ausentes 61

55 (56) Los aguijones del disco y cola, presentan costillas en parte de su recorrido, y son basalmente radiados o estrellados; 18 a 24 aguijones centrocaudales .57

56 (55) Los aguijones del disco y cola, no presentan costillas en su recorrido, siendo más bien lisos y de base oval; 12 a 30 aguijones centrocaudales. Dorso castaño oscuro, ventralmente blanco sucio con pe- queñas manchas negruzcas, borde del disco oscuro (Chile: Coquimbo al estrecho de Magallanes; Argentina: islas Malvinas; Uruguay).

Dipturus chilensis Guichenot, 1848.

57 (58) Más de 18 aguijones en la serie media...... 59

58 (57) Menos de 18 aguijones (usualmente 12 a 15) en la serie media, extendiéndose desde un punto en frente del ángulo posterior de la aleta pectoral a la primera aleta dorsal (Chile: estrecho de Magallanes; Argentina: Patagonia e islas Malvinas; Uruguay)

Amblyraja doello-juradoi Pozzi, 1935.

59 (60) Con 18 a 24 aguijones en la serie media (incluidos el nucal y escapular medio). De 1-2 aguijones interdorsales (Chile: Iquique al golfo de Ladrillero; Argentina; Uruguay) Amblyraja frerichsi Krefft, 1968.

60 (59) Usualmente con 20 a 28 (raramente 20 a lo más), aguijones en la serie media, que se extienden desde el nivel del ángulo posterior de las aletas pectorales hasta el origen de la primera aleta dorsal, de las cuales 4-6 se encuentran sobre el cuerpo hasta la axila de las aletas pectorales. Un aguijón nucal y un pequeño aguijón interdorsal (Talud del Mar de Ross en el Océano Austral; Chile: península Antártica e isla Georgia del Sur). Amblyraja georgiana Norman, 1938.

61 (62) Superficie ventral profusamente cubierta de abundante espinulación; sin aguijones escapulares ni supraescapulares (Chile: Los Vilos hasta San Pedro; sur de Argentina; Uruguay)

Dipturus trachyderma Krefft \& Stehmann, 1975.

62 (61) Superficie ventral lisa, o excepcionalmente espinulada sólo en la cola; 1 a 2 aguijones supraescapulares, y 1 aguijón escapular (Chile: Talcahuano a Corral; sur de Brasil)

Rajella sadowskii Krefft \& Stehmann, 1974.

63 (64) Fin de la base de la primera aleta dorsal notablemente por detrás del margen posterior del pliegue de la piel que une las bases de las aletas pélvicas con el cuerpo. Aletas pélvicas comienzan por detrás del margen posterior del disco o por detrás de éste. Distancia preoral menos que el ancho de la boca 65

64 (63) Fin de la base de la primera aleta dorsal claramente por encima del margen posterior del pliegue de la piel que une las bases de las aletas pélvicas 
con el cuerpo. Aletas pélvicas comienzan por encima del margen posterior del disco. Distancia preoral mayor o igual que el ancho de la boca (Chile: elevación de Nazca e isla Salas y Gómez)

... Torpedo semipelagica (Parin \& Kotlyar, 1985).

65 (66) Origen de las aletas pélvicas separadas del margen posterior del disco a una distancia no menor que la longitud basal de la primera aleta dorsal (Chile: elevación de Nazca e isla Salas y Gómez) .... Torpedo microdiscus (Parin \& Kotlyar, 1985).

66 (65) Origen de las aletas pélvicas por debajo del margen posterior del disco o separada de éste a una distancia no mayor que $1 / 3$ de la longitud basal de la primera aleta dorsal (Chile: Valparaíso, Lebu y

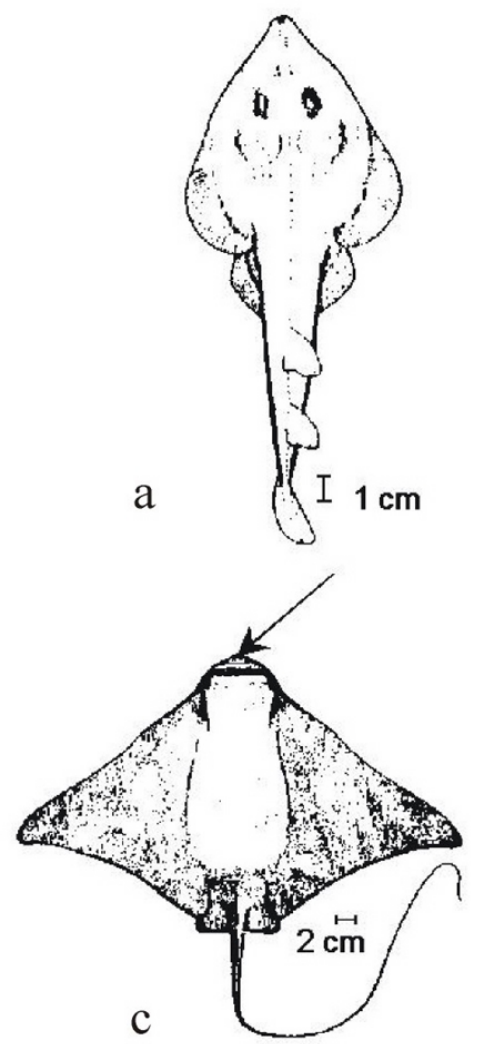

Valdivia) Torpedo tremens De Buen, 1959.

67 (68) Disco trapezoidal, márgenes anteriores convexos. Superficie dorsal del disco lisa en juveniles, una corrida de tubérculos mediodorsales en los adultos. Superficie dorsal y ventral del disco y pliegue ventral de la cola púrpura oscuro (Cosmopolita; en las cercanías de isla de Pascua)

Dasyatis violacea (Bonaparte, 1832).

68 (67) Disco subtriangular y redondeado, márgenes anteriores marcadamente convexo. Superficie del disco desnudo en juveniles, con 3 corridas de tubérculos sobre el dorso en adultos. Superficie dorsal oliva grisáceo y superficie ventral blanca a gris pálido (Desde California, Hawaii, Paita (Perú) has-

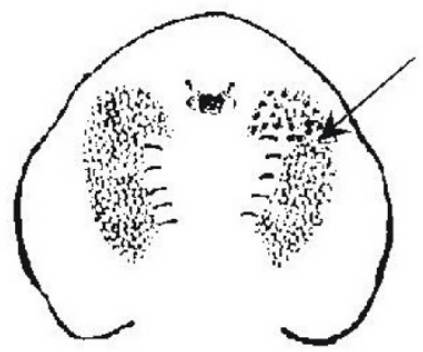

b

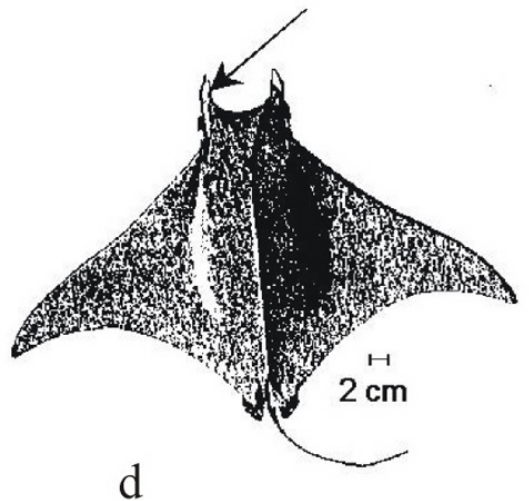

Figura 2. a) Esquema de la morfología externa de Rhinobatos planiceps Garman, 1880, b) posición y forma de los órganos eléctricos de una especie de la familia Torpedinidae (Vista ventral). La flecha señala la ubicación de estas estructuras, c) morfología de la cabeza en Myliobatidae: Myliobatis chilensis (Philippi, 1892): ausencia de aletas cefálicas, d) morfología de la cabeza en Mobulidae: Mobula tarapacana Garman, 1913: presencia de aletas cefálicas. La flecha señala la diferencia entre ambos taxa (modificado de Chirichigno, 1998).

Figure 2. a) External morphology scheme of Rhinobatos planiceps Garman, 1880, b) position and shape of electric organs in a species of Torpedinidae family. The arrow shows the position of these structures, c) head morphology in Myliobatidae: Myliobatis chilensis (Philippi, 1892): without cephalic fins, d) head morphology in Mobulidae: Mobula tarapacana Garman, 1913: presence of cephalic fins. The arrow shows the difference between both taxa (modified from Chirichigno, 1998). 
ta Antofagasta, Chile)

Dasyatis brevis (Garman, 1913).

69 (70) Superficie dorsal con vermiculaciones y reticulaciones muy distintivas; largo de la región caudal menor a la mitad del disco (Pacífico Sur: Chile) ....... Urobatis marmoratus (Philippi, 1892).

70 (69) Superficie dorsal con manchas y puntitos negros; largo de la región caudal igual o mayor al largo del disco (Nuevo México hasta Perú; Chile: en Quintero)

Urotrygon chilensis (Günther, 1871).

71 (72) Dorso del disco marrón-rojizo. Superficie ventral claro con extremos de las aletas pectorales oscuras y hocico con algunas manchas oscuras. Disco con mayor envergadura que longitud. Aleta rostral avanzada en visera de forma de cúpula ancha y corta, sencilla, no dividida, de bordes gruesos (Perú: Callao; Chile: golfo de Arauco a Valdivia)

.Myliobatis chilensis (Philippi, 1892).

72 (71) Dorso del disco café uniforme, más claro en los extremos laterales de las alas. Superficie ventral amarillenta sin márgenes oscuros. Disco amplio, agudo hacia los ángulos laterales, de extremidad roma. Aleta rostral saliente en visera, en forma de campana, con pequeño saliente medio (Perú: Paita; Chile: Arica, caleta Vitor a golfo de Arauco) . Myliobatis peruvianus Garman, 1913

73 (74) Espiráculo pequeño, subcircular, ventral al plano de las aletas pectorales. Láminas branquiales no fusionadas. Dientes grandes, teselados. Dentículos muy esparcidos o ausentes. Dorso café oscuro a verde oliváceo, vientre blanco anteriormente, gris posteriormente, con una irregular pero definida línea de demarcación (golfo de California y Chile) .......... Mobula tarapacana (Philippi, 1892).

74 (73) Espiráculos en una hendidura longitudinal alargada, dorsal al plano de las aletas pectorales. Láminas branquiales fusionadas. Dientes hexagonales, más ancho que largos. Dorso negro, ojos y parte dorsal de las aletas cefálicas negro grisáceo. Ventralmente blancuzco variando a gris hacia las puntas de las aletas pectorales. Piel desnuda, sin trazos de rugosidades o espinas (golfo de California y Chile)

Mobula thurstoni Beebe \& Tee-Van, 1941.

\section{GLOSARIO}

Aleta cefálica (o aleta rostral): prolongación carnosa en forma de paleta, presente en la cabeza de Mobulidae.

Aguijones: tipo de espinulación (escamas modificadas) presente en las rayas. Se caracterizan por ser evidentes visualmente, gruesas y punzantes, a diferencia de las espínulas. Según la ubicación de los aguijones sobre el cuerpo de la raya, va cambiando su nombre.

Boca protráctil: boca capaz de proyectarse hacia delante.

Cartílago rostral: en el neurocráneo de rayas, región cefálica que puede ser poco o muy prolongado.

Cortina nasal: alerones nasales derecho e izquierdos fusionados que dan una apariencia de cortina sobre el borde superior de la boca. Generalmente con bordes emarginados.

Dentículos: tipo de espinulación presente en las rayas. Se caracterizan por ser evidentes al tacto y tener forma de estrella.

Deprimido: aplastado dorsoventralmente.

Dientes hexagonales: tipos de dientes con seis bordes. Característico en Mobula thurstoni (Mobulidae).

Dientes en pavimento (o en mosaico): tipos de dientes que se caracterizan por ser aplanados, sin cúspides y disponerse en bandas muy juntas. Es característico en Discopyge tschudii (Narkidae).

Dientes teselados: tipos de dientes, que en su conjunto se asemejan a una peineta. Es característico en Mobula tarapacana (Mobulidae).

Disco: en rayas, superficie formada por la fusión de las aletas pectorales a la cabeza.

Emarginados: con flecos.

Espiráculo: abertura branquial que se extiende desde la cavidad faríngea, desde la cual emerge, justo detrás del ojo.

Fontanela anterior: abertura ubicada en el neurocráneo de los condrictios, entre las cápsulas nasales y que se encuentra cerrada por una membrana.

Fontanela nasal: abertura ubicada en las cápsulas nasales del neurocráneo de los condrictios. 
Tabla 1. Latitud y longitud de las localidades geográficas de las especies mencionadas en la clave.

Table 1. Latitude and longitude of geographic locations of the species cited in the key.

\begin{tabular}{|c|c|}
\hline Localidad & Coordenadas geográficas \\
\hline Islas Galápagos (Ecuador) & $03^{\circ} 15^{\prime} \mathrm{S}$ \\
\hline Huacho-Chancay (Perú) & $03^{\circ} 15^{\prime} \mathrm{S}-80^{\circ} 55^{\prime} \mathrm{W}$ \\
\hline Punta Pizarro (Perú) & $03^{\circ} 15^{\prime} \mathrm{S}-80^{\circ} 55^{\prime} \mathrm{W}$ \\
\hline Arica & $18^{\circ} 20^{\prime} \mathrm{S}$ \\
\hline Caleta Vitor & $18^{\circ} 44^{\prime} \mathrm{S}-70^{\circ} 19^{\prime} \mathrm{W}$ \\
\hline Punta Camarones & $19^{\circ} 12^{\prime} \mathrm{S}-70^{\circ} 17^{\prime} \mathrm{W}$ \\
\hline Iquique & $21^{\circ} 25^{\prime} \mathrm{S}$ \\
\hline Talcahuano & $22^{\circ} 30^{\prime} \mathrm{S}$ \\
\hline Hornitos, Antofagasta & $23^{\circ} 29^{\prime} \mathrm{S}-70^{\circ} 5^{\prime} \mathrm{W}$ \\
\hline La Portada, Antofagasta & $23^{\circ} 29^{\prime} \mathrm{S}-70^{\circ} 25^{\prime} \mathrm{W}$ \\
\hline Taltal & $25^{\circ} 25^{\prime} \mathrm{S}-70^{\circ} 35^{\prime} \mathrm{W}$ \\
\hline Isla Salas y Gómez & $26^{\circ} 27^{\prime} \mathrm{S}-105^{\circ} 21^{\prime} \mathrm{W}$ \\
\hline Isla de Pascua & $27^{\circ} 07^{\prime} \mathrm{S}-109^{\circ} 22^{\prime} \mathrm{W}$ \\
\hline Coquimbo & $29^{\circ} 50^{\prime} 7^{\prime \prime} \mathrm{S}-71^{\circ} 22^{\prime} \mathrm{W} ; 2^{\circ} 53^{\prime} \mathrm{S}-7^{\circ} 18^{\prime} \mathrm{W}$ \\
\hline Los Vilos & $31^{\circ} 55^{\prime} \mathrm{S}-71^{\circ} 31^{\prime} \mathrm{W}$ \\
\hline Pichidangui & $32^{\circ} 07^{\prime} 0^{\prime \prime} \mathrm{S}-71^{\circ} 35^{\prime} 5^{\prime \prime} \mathrm{W}$ \\
\hline Caleta la Ligua & $32^{\circ} 24^{\prime} 2^{\prime \prime} \mathrm{S}-71^{\circ} 28^{\prime} 0^{\prime \prime} \mathrm{W}$ \\
\hline Papudo & $32^{\circ} 31^{\prime} \mathrm{S}-71^{\circ} 27^{\prime} \mathrm{W}$ \\
\hline Valparaíso & $32^{\circ} 58^{\prime} \mathrm{S}-71^{\circ} 46^{\prime} \mathrm{W}$ \\
\hline Constitución & $35^{\circ} 17^{\prime} 3^{\prime \prime} \mathrm{S}-72^{\circ} 34^{\prime} 0^{\prime \prime} \mathrm{W}$ \\
\hline Golfo de Arauco & $37^{\circ} 22^{\prime} \mathrm{S}-86^{\circ} 27^{\prime} \mathrm{W}$ \\
\hline Lebu & $37^{\circ} 22^{\prime} \mathrm{S}-86^{\circ} 27^{\prime} \mathrm{W}$ \\
\hline Valdivia & $39^{\circ} 30^{\prime} \mathrm{S}-74^{\circ} 00^{\prime} \mathrm{W}$ \\
\hline Corral & $39^{\circ} 47^{\prime} 8^{\prime \prime} \mathrm{S}-73^{\circ} 34^{\prime} 0^{\prime \prime} \mathrm{W}$ \\
\hline Bahía Blanca & $40^{\circ} 22^{\prime} \mathrm{S}-59^{\circ} 14,7^{\prime} \mathrm{W}$ \\
\hline Bahía San Pedro & $41^{\circ} 04^{\prime} \mathrm{S}$ \\
\hline Ancud & $42^{\circ} 03^{\prime} 0^{\prime \prime} \mathrm{S}-73^{\circ} 11^{\prime} 0^{\prime \prime} \mathrm{W}$ \\
\hline Chiloé & $42^{\circ} 48^{\prime} \mathrm{S}-74^{\circ} 21^{\prime} \mathrm{W}$ \\
\hline Corcovado & $43^{\circ} 31^{\prime} \mathrm{S}-73^{\circ} 03^{\prime} \mathrm{W}$ \\
\hline Isla Guafo & $43^{\circ} 40^{\prime} \mathrm{S}-75^{\circ} 00^{\prime} \mathrm{W}$ \\
\hline Isla Guamblin & $45^{\circ} 04^{\prime} \mathrm{S}-75^{\circ} 27^{\prime} \mathrm{W}$ \\
\hline Canal Darwin & $45^{\circ} 22^{\prime} \mathrm{S}-75^{\circ} 29^{\prime} \mathrm{W}$ \\
\hline Archipiélago Los Chonos & $45^{\circ} 22^{\prime} \mathrm{S}-75^{\circ} 29^{\prime} \mathrm{W}$ \\
\hline Península de Taitao & $46^{\circ} 42,1^{\prime} \mathrm{S}-74^{\circ} 18^{\prime} \mathrm{W}$ \\
\hline Golfo Ladrillero & $49^{\circ} 01^{\prime} \mathrm{S}$ \\
\hline Canal de Concepción & $51^{\circ} 00^{\prime} \mathrm{S}-75^{\circ} 44^{\prime} \mathrm{W}$ \\
\hline Estrecho de Magallanes & $53^{\circ} 00^{\prime} \mathrm{S}-72^{\circ} 25^{\prime} \mathrm{W}$ \\
\hline Cabo de Hornos & $54^{\circ} 55^{\prime} \mathrm{S}-67^{\circ} 09^{\prime} \mathrm{W}$ \\
\hline Isla Diego Ramírez & $56^{\circ} 47^{\prime} \mathrm{S}-68^{\circ} 04^{\prime} \mathrm{W}$ \\
\hline Bahía de Samborombón (Argentina) & $35^{\circ} 43^{\prime} \mathrm{S}-52^{\circ} 43^{\prime} \mathrm{W}$ \\
\hline Islas Malvinas & $51^{\circ} 39^{\prime} \mathrm{S}-62^{\circ} 01^{\prime} \mathrm{W}$ \\
\hline Isla Los Estados (Argentina) & $54^{\circ} 55^{\prime} \mathrm{S}-67^{\circ} 09^{\prime} \mathrm{W}$ \\
\hline Sur de Brasil & $29^{\circ} 57^{\prime} \mathrm{S}-47^{\circ} 35^{\prime} \mathrm{W}$ \\
\hline
\end{tabular}


Hocico: porción de la cabeza la cual se proyecta más allá de los ojos.

Láminas branquiales: estructura carnosa, aplanada. Se localiza al interior de la branquia que puede tener laminillas branquiales que aumentan la superficie respiratoria. Las laminillas pueden fusionarse o no formando placas branquiales.

Piel lisa: piel carente de escamas.

Porción subcaudal: región localizada por debajo de la aleta caudal.

Radios pectorales: elementos cartilaginosos que sostienen a la aleta pectoral.

Tallo: sinónimo de pedúnculo caudal o cola.

\section{REFERENCIAS}

Bahamonde, N. \& G. Pequeño. 1975. Peces de Chile. Lista Sistemática. Mus. Nac. Hist. Nat., Chile, Publ. Ocas., 21: 3-20.

Beebe, W. \& J. Tee-Van. 1941. Fishes from the Tropical Eastern Pacific. Rays, mantas and chimaeras. Zoologica, N.Y., 26(3): 245-280.

Bigelow, H.B. \& W.C. Schroeder. 1965. Notes on a small collection of rajids from the subantarctic region. Limnol. Oceanogr. (Suppl. to V), 10: R38R49.

Chirichigno, F.N. 1969. Lista sistemática de los peces marinos comunes para Ecuador-Perú-Chile. Comisión Permanente del Pacífico Sur, 108 pp.

Chirichigno, F.N. 1974. Clave para identificar los peces marinos del Perú. Inf. Inst. Mar Perú-Callao, 44: 1-387.

Chirichigno, F.N. 1978. Nuevas adiciones a la ictiofauna marina del Perú. Inf. Inst. Mar. PerúCallao, 46: 1-109.

Chirichigno, F.N. 1998. Clave para identificar los peces marinos del Perú. Publ. Esp. Inst. Mar. PerúCallao, 302 pp.

Chirichigno, F.N. \& J.E. McEachran. 1979. Urolophus tumbensis, a new stingray from the coast of Perú (Batoidea; Urolophidae). Copeia, 1979(4): 709-713.

Chu, Y.T., Q.W. Meng, A.S. Hu \& S. Li. 1981. Description of four new species, a new genus and a new family of elasmobranchiate fishes from deepsea of the South China Sea. Oceanol. Limnol. Sin., 12(2): 103-116.

Compagno, L.J.V. 1973. Interrrelationships of living elasmobranchs. En: P.H. Greenwood, R.S. Miles \& C. Patterson (eds.). Interrelationships of fishes. J. Linn. Soc. (Zool.), 53(Suppl. 1): 1-37.

Compagno, L.J.V. 1977. Phyletic relationships of living sharks and rays. Amer. Zool., 17(2): 302-322.

De Buen, F. 1959a. Notas sobre ictiología chilena, con descripción de dos nuevas especies. Rev. Biol. Mar., Valparaíso, 9(1-3): 257-270.

De Buen, F. 1959b. Notas preliminares sobre la fauna marina preabismal de Chile, con descripción de una familia de rayas, dos géneros y siete especies nuevas. Bol. Mus. Nac. Hist. Nat., Chile, 27(3): 171202.

De Buen, F. 1959c. Lampreas, tiburones, rayas y peces en la Estación de Biología Marina de Montemar, Chile. Rev. Biol. Mar., Valparaíso, 9(13): 3-200.

De Buen, F. 1960. Tiburones, rayas y quimeras en la Estación de Biología Marina de Montemar, Chile. Rev. Biol. Mar., Valparaíso, 10(1-3): 1-50.

Eschmayer, W. 1998. Catalog of Fishes. Cal. Acad. Sci.. San Francisco. http//: www. calacademy.org

Fowler, H.W. 1945. Fishes of Chile, Systematic Catalog. Apartado Rev. Chil. Hist. Nat., Parts I and II, pp. 36-171.

Guichenot, A. 1848. Peces. En: C. Gay (ed.). Historia Física y Política de Chile. De Maule et Renou, París, 372 pp.

Henríquez, G., L. Rodríguez \& I. Kong. 1981. Exploración y prospección de recursos pesqueros del talud continental. Corporación de Fomento de Producción. Instituto de Fomento Pesquero, AP81/5: 14-48 pp.

Hubbs, L. \& R. Ishiyama. 1968. Methods for the taxonomy study and description of skates (Rajidae). Copeia, 1968(3): 483-491.

Hulley, P.A. 1970. An investigation of the Rajidae of the west and south coasts of southern Africa. Ann. South Afr. Mus., 55(4): 151-220.

Hulley, P.A. 1972. The origin, interrelationships and distribution of South African Rajidae 
(Chondrichthyes, Batoidei). Ann. South Afr. Mus., 60(1): 1-103.

Hulley, P.A. \& M. Stehmann. 1977. The vality of Malacoraja Stehmann, 1970 (Chondrichthyes, Batoidei, Rajidae) and its phylogenetic significance. Ann. South African Mus., 72(12): 227-237.

Hureau, J.C. 1966. Nouvelle description de Raja eatoni Günther 1879, Rajidae endémique des Iles Kerguelen (Antarctique). Bull. Mus. Nat. Hist. Nat., Paris, 2é ser., 38(4): 396-399.

Ishihara, H. 1990. The skates and rays of the wester North Pacific an overview of their fisheries utilization and classification. En: H.L Pratt Jr., S.H. Gruber \& T. Taniuchi (eds.). Elasmobranches as living resources: Advances in the biology, ecology, systematics and the status of the fisheries, NOAA Tech. Rep. 90, Tokyo, pp. 485-497.

Ishihara, H. \& R. Ishiyama. 1986. Systematic and distribution of the skates of the North Pacific (Chondrichthyes, Rajoidei). En: T. Uyeno, R. Arai, T. Taniuchi \& K. Matsuura (eds.). Indo-Pacific Fish Biology: Proceedings of the Second International Conference on Indo-Pacific Fishes. Ichthyol. Soc. Japan, Tokyo, pp. 269-280.

Ishiyama, R. 1958. Studies on the rajid fishes (Rajidae) found in the waters around Japan. J. Shimonosehi Coll. Fish., 7: 193-394.

Ishiyama, R. \& C.L. Hubbs. 1968. Bathyraja a genus of Pacific skates (Rajidae) regarded as phyletically distinct from the Atlantic genus Breviraja. Copeia, 1968(2): 407-410.

Krefft, G. 1968. Neue un erstmalig nachgewiesene Knorpelfische aus dem Archibental des Südwestatlantiks, einschliesslich einer Diskussion einiger Etmopterus. Arten südlicher Meer. Archiv. Fisc., 19(1): 1-42.

Lamilla, J. 1986. Bathyraja albomaculata (Norman,1937): Primer registro para las costas de Chile (Chondrichtyes, Rajidae). Bol. Soc. Biol. Concepción, 57: 177-182.

Lamilla, J. \& R. Meléndez. 1989. Primer registro de Dasyatis violacea (Bonaparte 1832) (Myliobatiformes: Dasyatidae), en las cercanías de Isla de Pascua. Estud. Oceanol., 8: 45-50.

Lamilla, J., G. Pequeño \& H. Figueroa. 1984. Aspectos biológicos de Psammobatis lima, Poeppig,
1835 , en el litoral de Valdivia, Chile (Elasmobranchii, Rajidae). Rev. Com. Perm. Pacífico Sur, 14: 183-209.

Lamilla, J. \& G. Pequeño. 1999. Descripción esquelética comparativa y primer registro en el océano Pacífico de Bathyraja multispinis (Norman, 1937) (Rajiformes; Rajoidei), con referencia a otras especies de Bathyraja. Rev. Biol. Mar. Oceanogr., 34(2): 281-290.

Lamilla, J., G. Pequeño \& I. Kong. 1995. Dasyatis brevis (Garman, 1880) segunda especie de Dasyatidae registrada para Chile (Chondrichthyes, Myliobatiformes). Invest. Oceanol., 14: 23-27.

Leible, M. 1987. Descripción taxonómica de juveniles y adultos de Raja (Dipturus) flavirostris Philippi, 1892 (Rajiformes, Rajidae), capturados frente a la costa de Chile Central. Gayana, Zool., 51(1-4): 131-176.

Leible, M. 1988. Revisión de métodos para estudios taxonómicos de rayas (Rajiformes, Rajidae). Gayana, Zool., 52(1-2): 15-93.

Leible, M., J. Carvajal \& M. Fuentealba. 1990. Polimorfismo en Raja (Dipturus) flavirostris Philippi, 1892: Análisis morfológico y parasitario. Bol. Soc. Biol. Concepción, 61: 93-102.

Leible, M. \& M. Sthemann. 1987. First record of Raja (Dipturus) trachyderma Kreft y Stehmann, 1975 from the Southeastern Pacific off Chile, with first description of its clasper characters and additional skeletal morphological details (Pisces, Rajiformes, Rajidae). Stud. Neotr. Fauna and Env., 22(4): 169-188.

Mann, G. 1954. La vida de los peces en aguas chilenas. Instituto de Investigaciones Veterinarias y Universidad de Chile, Santiago 342 pp.

McEachran, J.D. 1982a. Revision of the Southamerican skate genus Sympterygia (Elasmobranchii, Rajiformes). Copeia, 1982(4): 867-890.

McEachran, J.D. 1982b. Chondrichthyes. En: P.S. Parker (ed.). Synopsis and classification of living organisms. McGraw-Hill Co., pp. 831-858.

McEachran, J.D. 1983. Results of the research cruises of FRV "Walther Herwig" to South America. LXI. Revision of the Souh American skate genus Psammobatis Günther 1870 (Elasmobranchii; Rajiformes, Rajidae). Arch. Fisch. Wiss., 34(1): 23 80. 
McEachran, J.D. 1984. Anatomical investigations of the New Zeland skates Bathyraja asperula and $B$. spinifera, with an evalutaion of their classification within the Rajoidei (Chondrichthyes). Copeia, 1984 (1): 45-58.

McEachran, J.D. 1985. Urolophidae. Rayas redondas. En: W. Fischer, F. Krupp, W. Schneider, C. Sommer, K.E. Carpenter \& V. Niem (eds.). Guía FAO para identificación de especies para los fines de la pesca. Pacífico Centro-Oriental, FAO, Rome, 3 vols.

McEachran, J.D. \& L.J.V. Compagno. 1979. A further description of Gurgesiella furvescens with comments of the interrelationships of Gurgesiellidae and Pseudorajidae (Pisces, Rajoidei). Bull. Mar. Sci., 29(4): 530-553.

McEachran, J.D. \& L.J.V. Compagno. 1982. Interrrelationships of and within Breviraja based on anatomical structures (Pisces: Rajoidei). Bull. Mar. Sci., 32(2): 399-425.

McEachran, J.D. \& K.A. Dunn. 1998. Phylogenetic analysis of skates, a morphologically conservative clade of Elasmobranchs (Chondrichthyes: Rajidae). Copeia, 1998 (2): 271-290.

McEachran, J.D. \& R.E. Matheseon, Jr. 1985. Polychromatism and polymorphism in Breviraja spinosa (Elasmobranchii, Rajiformes), with description of three new species. Copeia, 1985(4): 1035-1052.

McEachran, J.D. \& T. Miyake. 1984. Comments on the skates of the tropical eastern Pacific: One new species and three new records (Elasmobranchii: Rajiformes). Proc. Biol. Soc. Wash., 97(4): 773787.

McEachran, J.D. \& T. Miyake. 1986. Interrelationships within a putative monophyletic group of skates (Chondrichthyes Rajoidei, Rajini). Indo Pacific fish biology. En: T. Uyeno, R. Arai, T. Taniuchi \& K. Matsukura (eds.). Proceedings of the Second International Conference on Indo Pacific Fishes, conducted at the Tokyo National Museum, Uyeno Park, Tokyo, pp. 281-290.

McEachran, J.D. \& T. Miyake. 1987. A new species of skate of the genus Breviraja from off Nova Scotia, with comments on the status of Breviraja and Neoraja (Chondrichthyes, Rajoidei). Copeia, 2: 409-417.

McEachran, J.D. \& T. Miyake. 1990. Phylogenetic interrelationships of skates: a working hypothesis (Chondrichthyes, Rajoidei). NOAA, Tech. Rep. NMFS 90: 258-304.

Menni, R.C. 1971. Anatomía del myxopterigio y posición sistemática de Raja flavirostris Philippi 1892 (Chondrichthyes, Rajiformes). Neotropica, 17(52): 39-43.

Menni, R.C. 1972a. Anatomía del myxopterigio y diferencias en los géneros Psammobatis y Sympterygia (Chondrichthyes, Rajidae). Neotropica, 18(56): 73-80.

Menni, R.C. 1972b. «Raja» (Atlantoraja), subgen. nov. y lista crítica de los «Rajidae» argentinos (Chondrichthyes, Rajiformes). Rev. Mus. de La Plata, Zool., 11: 165-173.

Menni, R.C. 1973. Rajidae del litoral bonaerense. I Especies de los géneros Raja, Bathyraja y Sympterygia (Chondrichthyes). Physis, Sec. A., 32(85): 413-439.

Nelson, J.S. 1994. Fishes of the world. John Wiley \& Sons, New York, 600 pp.

Nishida, K. 1990. Phylogeny of the suborder Myliobatidoidei. Mem., Fac. Fish. Hokkaido Univ., 37(1-2): 1-108.

Norman, J.R. 1935. Coast fishes. Part 1. The south Atlantic. Discovery Reps., 12: 1-58.

Norman, J.R. 1937. Coast fishes. Part 2. The Patagonian region. Discovery Reps., 16: 1-150.

Notarbartolo di Sciara, G. 1987. A revisionary study of the genus Mobula Rafinesque, 1810 (Chondrichthyes: Mobulidae) with the description of a new species. Zool. J. Linn. Soc., 91(1): 1-91.

Oliver, S.C. 1943. Catálogo de los peces marinos del litoral de Concepción y Arauco. Bol. Soc. Biol. Concepción, 17: 75-126.

Parin, N.V., G.A. Golovan, N.P. Pakhorukov, Y.I. Sazonov \& Y.N. Shcherbachev. 1980. Fishes from the Nazca and Sala y Gómez underwater ridges collected in cruise of R/V "Ikhtiandr". En: N.V. Parin (ed.). Fishes of the open ocean. Acad. Sci. USRR; PP. Shirshov Inst. Oceanol., Moscow, pp. 5-18.

Parin, N.V. \& A.N. Kotlyar. 1985. Las rayas del género Torpedo en los mares abiertos del Pacífico sur oriental. Vopr. Ikhtiol., 25: 707-718 (en ruso). 
Pequeño, G. 1975a. Peces del crucero Merluza V efectuado con el B/C "Carlos Darwin" entre Corral y Coquimbo. Enero-Febrero de 1970. Bol. Mus. Nac. Hist. Nat., Chile, 34: 227-232.

Pequeño, G. 1975b. Nuevo registro de Myliobatis chilensis Philippi (Elasmobranchii, Myliobatidae). Bol. Soc. Biol. Concepción, 49: 57-160.

Pequeño, G. 1989. Peces de Chile. Lista sistemática revisada y comentada. Rev. Biol. Mar., Valparaíso, 24: 1-132.

Pequeño, G. 1997. Peces de Chile. Lista sistemática revisada y comentada: Addendum. Rev. Biol. Mar. y Oceanogr., Valparaíso, 32(2): 77-94.

Pequeño, G. \& J. Lamilla. 1979. Primer hallazgo de una hembra de Gurgesiella furvescens De Buen 1959 (Elasmobranchii, Gurgesiellidae). An. Mus. Hist. Nat., Valparaíso, 12: 233-237.

Pequeño, G. \& J. Lamilla. 1985. Estudio sobre una colección de rayas del sur de Chile (Chondrichthyes, Rajidae). Rev. Biol. Mar., Valparaíso, 21(2): 225-271.

Pequeño, G. \& J. Lamilla. 1993. Batoídeos comunes a las costas de Chile y Argentina-Uruguay (Pisces: Chondrichthyes). Rev. Biol. Mar., Valparaíso, 28(2): 203-217.

Pequeño, G. \& J. Lamilla. 1996. Preliminary report on the demersal fish material collected during the «Victor Hensen» Magellan Campaign. Ver. zur Polarfosch., 190: 68-70; 105-106.

Recibido: 13 enero 2003; Aceptado: 17 junio 2003
Philippi, R. A. 1857a. Raja scobina. An. U. de Chile, 14: $1-187$.

Philippi, R.A. 1857b. Rhynchobatys Ph. nuevo género de rayas. An. U. de Chile, 14: 207-208.

Springer, S. 1971. Three species of skates (Rajidae) from the continental waters of Antarctica. Biology of the Antarctic Seas IV. Antarct. Res. Ser., 17: 110.

Stehmann, M. 1970. Vergleichend morphologische und anatomische Untersuchungen zur Neueordnung der Systematik der nordosatlantischen Rajidae (Chondrichthyes, Batoidei). Archiv Fisch. Wiss., 21(2): 73-164.

Stehmann, M. 1979. Illustrated field guide to abundant marine fish species in Argentina waters. Mitteilungen aus dem Inst. Seefisch, Hamburg, 23: $1-153$.

Stehmann, M. 1985. Batoid fishes. En: W. Fischer \& J.C. Hureau (eds.). FAO species identification sheets for Fishery Purposes, Southern Ocean, pp. 217-232.

Stehmann, M. 1986. Notes on the systematics of the rajid genus Bathyraja and its distribution in the world oceans. En: T. Uyeno, R. Arai, T. Taniuchi \& K. Matsuura. (eds). Indo Pacific Fishes. Ichthyol. Soc. Japan, Tokyo, pp. 261-268.

Zorzi, G.D. y M.E. Anderson. 1988. Records of the deep-sea skates, Raja (Amblyraja) badia Garman, 1899 and Bathyraja abyssicola (Gilbert, 1896) in the eastern north Pacific, with a new key to California skates. Calif. Fish Game, 74(2): 87-105. 